\title{
Investigation on scour protection of submarine piggyback pipeline
}

\author{
Shaopeng Yang ${ }^{1}$, Bing Shi $^{1}$,Yakun Guo ${ }^{2 *}$, Lipeng Yang ${ }^{3}$ \\ 1.College of Engineering, Ocean University of China,Qingdao,266100, China \\ 2.Faculty of Engineering\& Informatics, University of Bradford, BD7 1DP, UK \\ 3. The Administrative Committee of Qingdao Dongjiakou Economic Zone, 266400, China
}

\section{Highlights}

- A new type of piggyback pipeline structure is proposed

- New type of piggyback pipeline can reduce the depth and width of scour hole around pipeline

- Little scour around this new type piggyback pipeline occurs when small pipe diameter over large pipe diameter over a certain value

\begin{abstract}
This paper presents the results of laboratory experiments and numerical simulations to investigate the effect of different piggyback pipeline configuration on the morphology of local seabed scour subject to steady currents. Piggyback pipeline configuration investigated includes the commonly used piggyback pipeline, namely a small pipe attached on the top of large pipe and new form of piggyback pipeline proposed in this study in which a small pipe is attached to the large pipe on the upstream and downstream side, respectively. Pressure gradient, drag coefficient, lift coefficient and scour extent around pipelines are measured and analyzed for a range of pipelines and current conditions. Results show that the vortex strength downstream of the commonly used piggyback
\end{abstract}

${ }^{*}$ Corresponding author: Yakun Guo (E-mail address: y.guo16@bradford.ac.uk) 
pipeline is larger than that for a single as well as the new piggyback pipeline under the same condition. This new type piggyback pipeline can effectively reduce the depth and width of the scour hole. In particular, when the ratio of the small pipe diameter over the large pipe diameter is greater than 0.3 , little scour under this new type piggyback pipeline occurs for the test conditions. The bed topography downstream of the pipe has also been altered to favor the backfill.

Keywords: piggyback pipeline; stead current; diameter ratio; scour hole; scour protection

\section{Introduction}

In recent years, as a new-model of offshore oil and gas transport pipeline, the piggyback pipeline has been gradually used in offshore oil and gas engineering due to its low design and construction costs as well as short construction period (Brockbank, 1990). The most general configuration of piggyback pipeline bundles comprises one large pipeline with a small pipe attached directly above the large one, as shown in Fig.1 (a). The large pipe transports the oil and gas, while the small pipe transports monitoring signal and the oil displacement material (Jakobsen and Sayer, 1995). This pipeline has been adopted and applied to the marginal oilfield development in the Bohai Sea of China (Yang et al., 2007).

When a structure (e.g. pipeline, mono-pile) is installed in offshore seabed, the structure will be subjected to wave or current or the combination of wave and current loading (Sumer 2014; Fredsøe 2016; Lin et al. 2017; Sui et al. 2017). The structure in turn generates a complex wave or currentstructure-seabed interaction system (Sumer 2014; Fredsøe 2016; Sui et al. 2016). Though extensive studies have been conducted to investigate the marine loading (wave and/or current) acting on a single pipeline laying or embedded in seabed (e.g. MacPherson 1978; Sudhan et al. 2002; Lin et al. 
2016; Fredsøe 2016; Sun et al. 2019) and the local scour depth beneath the pipeline in clear-water and live-bed conditions (Mao 1986; Sumer et al. 2001; Najafzadeh et al. 2014a,b; Najafzadeh and Sarkamaryan 2018; Najafzadeh and Saberi-Movahed 2018), relatively few studies have been conducted to investigate the marine waves and/or currents interacting with a piggyback pipeline. Similar to single pipeline, most piggyback pipelines are directly exposed to the marine environment and are subject to several marine environmental loads, such as underwater currents and waves. In addition, due to its complex structure and the interference between the two pipes, the piggyback pipeline is more vulnerable to damage than the single pipe (Kalghatgi and Sayer, 1997).This means that further studies are required to improve the design of piggyback pipeline and thus its stability.

Ma and Wang (1993) investigated the hydrodynamic loading under the action of waves and current on the piggyback pipeline. Their results showed that the drag coefficient $C_{D}$ and the inertia coefficient $C_{M}$ decreased with the increase of the Keulegan-Carpenter $(K C)$ number. They also found that $C_{M}$ varied between 1 4. Jester and Kallinderis (2003), Liu et al. (2007), Lee and Yang (2009) investigated the incompressible flow interacting with the fixed cylinder pairs of various arrangements, including tandem, side-by-side and staggered. They classified the flow regimes into different types based on the streamlines and contours of span wise vorticity. Using a Computational Fluid Dynamics (CFD) model, Kamarudin et al. (2006) investigated the hydrodynamic loading on the bundle under steady flow condition. Their results showed that the angle of the small pipe had great influence on the drag and lift coefficient for the bundle. They also showed that the equivalent diameter approach might underestimate the force acting on the bundle. Zhao and Cheng $(2007 ; 2008)$ and Zhao (2012) applied numerical models to investigate the vortex shedding and the hydrodynamic force acting on the piggyback pipeline. Their results demonstrated that the vortex shedding always 
existed when the ratio $(G / D)$ of the gap $G$ over the larger pipeline diameter $D$ (see Fig. 1(a)) was greater than 0.6 ; while the vortex was suppressed when $G / D \leqslant 0.2$. For very small ratio (e.g. $G / D<0.15$ ), only one vortex appeared downstream. While when $G / D \geqslant 0.15$, there were two vortices occurred in the lee side of the piggyback pipeline. They also found that the scour depth under the piggyback pipeline center reached the maximum value when the gap ratio $G / D=0.15$. Study of Cheng et al. (2013) showed that the hydrodynamic coefficient decreased with the increase of incoming flow velocity. They found that the positive lift coefficient $C_{L}{ }^{+}$decreased with the increase of $e / D$ ( $e$ is the gap between the bottom of the large pipe and the seabed, see Fig. 1(a)) and with the decrease of $G / D$ while $\mid C_{L}^{-}$|increased with the increase of $e / D$ and decreased with the increase of $G / D$. Zang et al. (2013) investigated the vortex induced vibration (VIV) of the piggyback pipeline. The asymmetry of the transverse vibration configuration for the piggyback pipeline was observed in their study, especially for the ratio of the gap over the diameter ranging from 0.1 to 0.5 . Cheng et al. (2012) investigated the influence of the hydrodynamic wave loading on the piggyback pipeline under wave action. Their results indicated that the force coefficients initially decreased and then remained almost constant when $e / D$ was beyond 0.5 . Zang and Gao (2014) carried out the physical modeling studies to investigate the influence of the mass-damping parameter, the ratio of between the gap and large pipe diameter, the ratio of the spacing over the large pipe diameter and the position angle of small pipe on the VIV response. In their tests, they found that for the small pipe placed directly above the main large pipe (namely $\theta=90^{\circ}$ ), the VIV was suppressed most effectively by the small pipe at $G / D \approx 0.25$. For $G / D=0.25$, the minimum peak amplitude and the maximum critical reduced velocity occurred at the position of $\theta \approx 120^{\circ}$. Using a numerical model, Panet al. (2015) investigated the VIV characteristics of the piggyback pipeline. Their numerical results revealed that 
the drag force of the piggyback pipeline was about $65 \%$ higher than that of a single pipe. Local scour around the piggyback pipeline under steady current has been studied by Zhao et al. (2018) who applied both the numerical simulation and physical experiment. They found that the scour depth has been significantly influenced by the inflow Re number and the gap-ratio. Analysis of the results showed that the scour depth increased with the increase of the inflow Re number and decreased with the increase of the gap-ratio.

Though the above studies have demonstrated some features of current/wave interacting with the typical piggyback pipeline, there still exists some challenge and the problem remains far from fully understood. For example, relatively few studies on the scour scale of the piggyback pipeline have been carried out though Mao (1986); Cevik and Yüksel (1999); Sumer et al. (2001) and Yang et al. (2012a, b; 2014) investigated the scour around a single pipe subject to constant flow or wave action. For the protection of the scour around the piggyback pipeline, even fewer studies have been performed to investigate the problem due to the complicated hydrodynamics and vortex-inducedvibration (VIV), which motivates this study. In this paper, a serious of laboratory flume experiment is performed to investigate the scour around the piggyback pipeline. Stimulated by Mao's study (1986) in which the onset scour was caused by the sediment piping induced by the vortex generated pressure difference between upstream and downstream of pipe, this study considers how to reduce the pressure gradient between the upstream and downstream sides of the main pipe. To this end, the configuration of the typical piggyback pipeline is modified accordingly, as shown in Fig.1 (b). In order to verify the rationality of this piggyback configuration, numerical simulation and laboratory experiments are carried out to investigate the flow, pressure field, sediment transport and scour around the pipeline. 


\section{Laboratory experiment and numerical simulation}

\subsection{Experimental set up}

The laboratory experiments are carried out in a wave flume in Ocean University of China. The wave flume is $25 \mathrm{~m}$ in length, $0.5 \mathrm{~m}$ in width and $0.6 \mathrm{~m}$ in depth. The pipes, having the same width as that of the flume, are placed on the sandy bed, as shown in Fig.2 (a) (plan view). A $0.15 \mathrm{~m}$ high and $5 \mathrm{~m}$ long sandy bed is built in the middle of the flume, with 1:10 slope at each end, as shown in Fig. 2 (b) (side view).Twelve water proof force transducers are installed on the main pipe surface to measure the pressure field around the piggyback pipelines, as shown in Fig.2 (c). Fig. 3 shows the force transducer (a) and Acoustic Doppler Velocimeter (b) used in the experiments for the measurement of pressure and flow field.

The main pipe tested has the outer diameter $(D)$ of $0.08 \mathrm{~m}, 0.10 \mathrm{~m}, 0.12 \mathrm{~m}$ and the small pipe diameters $(d)$ tested are $0.008 \mathrm{~m}, 0.01 \mathrm{~m}, 0.012 \mathrm{~m}, 0.016 \mathrm{~m}, 0.02 \mathrm{~m}, 0.024 \mathrm{~m}, 0.025 \mathrm{~m}, 0.03 \mathrm{~m} .0 .036 \mathrm{~m}$, respectively. In order to investigate the effect of current velocities on the local scour around the piggyback pipeline, three inflow velocities $0.2 \mathrm{~m} / \mathrm{s}, 0.25 \mathrm{~m} / \mathrm{s}$ and $0.32 \mathrm{~m} / \mathrm{s}$ are tested. The flow velocity is measured using an ADV (see Fig. 3(b)), which is mounted on a rail along the flume. The water temperature is measured using a thermometer and is kept as constant of $20^{\circ} \mathrm{C}$. The water depth is kept at a constant of $0.4 \mathrm{~m}$ for all tests.

In practical applications, the diameter of the submarine pipeline is usually between $0.3 \sim 4 \mathrm{~m}$. This experimental test is mainly for the moderate pipeline size of $1 \sim 3 \mathrm{~m}$. Therefore, the corresponding model scale for the pipeline size is about $1 / 10 \sim 1 / 20$. In general, based on most laboratory model scale, the test sand would be silt or clay. As silt or clay is cohesive and has strong 
flocculation and is not suitable for simulating the characteristics of seabed sediment, the experimental test adopts the prototype sand. The mean diameter of tested sand is $d_{50}=0.2 \mathrm{~mm}$, the specific gravity of the tested sand is $2650 \mathrm{~kg} / \mathrm{m}^{3}$ and the porosity of the tested sand is $\mathrm{n}=0.4$.The particle size distribution of sediment used in experiments is shown in Fig.4.

For the tested sediment, the value for the Shields parameter can be calculated by (Chien and Wan 1999):

$$
\theta=\frac{\tau_{\mathrm{c}}}{\left(\rho_{s}-\rho\right) g d_{s}}
$$

Where $\tau_{\mathrm{c}}$ is the shear force, $\rho_{s}$ is the density of sediment, $\rho$ is the density of the water and $d_{s}$ is the mean particle size of sand (taken as $d_{50}$ ).

The shear force is defined as

$$
\tau_{\mathrm{c}}=\rho v_{*}^{2}
$$

where $v *$ is the friction velocity which can be obtained using the logarithmic velocity distribution formula (Einstein and Chien 1955)

$$
\frac{v}{v_{*}^{2}}=5.75 \lg \left(30.2 \frac{y x}{K_{s}}\right)
$$

where $v$ is the velocity of flow, $K_{s}$ is the roughness of bed and can be replaced by $d_{65}=0.27 \mathrm{~mm}$ (from Fig. 4), $x$ is the correction parameters, $x=f\left(K_{s} / \delta\right), \delta$ is the thickness of the near-wall flow, $\delta=11.6 v / v_{*} \quad v$ is the corresponding kinematic viscosity, $\mathrm{y}$ is the height of the velocity measurement point, which can be taken as $0.5 D$.

Substituting $v=0.2 \mathrm{~m} / \mathrm{s}, 0.25 \mathrm{~m} / \mathrm{s}$ and $0.32 \mathrm{~m} / \mathrm{s}$ into the above equation respectively, $\theta=0.026$, 0.0416 and 0.0682 can be obtained. According to the shield curve, when $\theta=0.026$ and 0.0416 , it is clear water conditions, when $\theta=0.0682$, it is live bed scour. This means that for the chosen 
experimental velocity $v=0.2 \mathrm{~m} / \mathrm{s}, 0.25 \mathrm{~m} / \mathrm{s}$, the scour will be in clear water, while for $v=0.32 \mathrm{~m} / \mathrm{s}$, it will be in live bed scour.

Each experimental run lasts for 4 hours. The scour depth and scour profile are monitored at regular intervals until the scour reaches the quasi-equilibrium state. The quasi-equilibrium scour profiles are measured using a depth probes installed on rail along the flume with a least reading of $0.1 \mathrm{~mm}$. Table 1 lists the experimental parameters. In total 45 experiments are carried out in which 9 in Run 1, 12 in Run 2, Run 3 and Run 4, respectively.

\subsection{Numerical simulation}

The initial pressure distribution and the flow field near the single main pipeline, commonly used piggyback pipeline and new configuration of the piggyback pipeline under the action of constant flow are simulated using the numerical method of Zhang et al. (2013). A rectangular computational domain with $6 \mathrm{~m}$ in length and $0.4 \mathrm{~m}$ in width is used in the simulation. In order to simulate scoring process below the new piggyback pipeline, a two dimensional numerical model of local scour based on incompressible Reynolds-averaged Navier-Stokes (RANS) equations, the continuity equation and finite volume method is established through the redevelopment in FLUENT code. Dynamic mesh is used to capture the change of bed surface profile. $k$ - $\omega$ turbulence closure model is used for turbulence simulation to better simulate the near bed flow (Wilcox 2008). The calculated parameters of the bed are computed via UDF (user defined function). The free surface between water and air has been resolved by the volume of fluid (VOF) method. The left inlet of the computational domain is set as the velocity inlet and at the outlet the pressure outlet boundary is applied (Guo et al. 2008, 2014). The water surface boundary is specified as the atmospheric 
pressure boundary while the bed boundary is set as the wall boundary. The velocity at the first mesh near the bed is estimated using the standard wall function law (Launder and Spalding 1974). The initial conditions of the hydrodynamic pressure and surface displacements are set to zero. Nonuniform unstructured meshes are used around the new piggyback pipeline to better fit the shape of the pipeline. This mesh arrangement has advantage of locally refining the concerned regions, such as the pipeline area (Guo et al. 2012; Jing et al. 2009, 2011). The mesh densities in all the computations are the same as those shown in Fig. 5 in which $\mathrm{D}=0.1 \mathrm{~m}$ and $\mathrm{d}=0.03 \mathrm{~m}$. The total nodal point number is 63983 with the minimum mesh size being of about $0.0005 \mathrm{~m}$. The relationship between numerical results and mesh density is tested for convergence which shows that the numerical results have little change when the mesh nodal point is more than 63983 . This mesh density is therefore used for all simulations. The dimensionless time step of 0.001 is used in all the computations. The diameters of small and large pipe and the water depth are set as the same as those used in the experiments.

Six vertical velocity profiles from the numerical simulation are used to compare the flow field difference between upstream and downstream side of the pipeline for various pipeline arrangements. The locations of these velocity profiles chosen are shown in Fig.6.

\section{Results and Discussion}

\subsection{Experimental Observations}

Figure 7 shows the experimental photos of the scour around the pipe at the quasi-equilibrium state for common piggyback pipeline (Fig.7(a)) and new form piggyback pipeline with different diameter ratios for $\theta=0.0682(v=0.32 \mathrm{~m} / \mathrm{s})$. It can be seen from Fig.7 that the depth of scour hole 
changes when the position and size of the small pipe changes .As shown in Fig.7(b), (c), (d) and (e) for the new piggyback pipeline, the quasi-equilibrium scour depth is reduced when the ratio of small pipe diameter over the large pipe diameter increases. Fig. 7(d) demonstrates that when the smalllarge pipeline diameter ratio increases to 0.25 , the quasi-equilibrium scour depth is significantly reduced. When the diameter ratio is beyond the critical value of 0.3 , little scour depth is generated. Fig.7 (e) also shows that the bed elevation downstream of the pipe has risen, which may favor the backfill.

Figure 8 displays the variation of the scour depth $(h)$ with time $(t)$ for $D=0.1 \mathrm{~m}$ and $d=0.01 \mathrm{~m}$ and $\theta=0.0682(\nu=0.32 \mathrm{~m} / \mathrm{s})$. It is seen that the scour depth has as sharp increase during the first two hours of the experiment. The scour depth then gradually develops. There is a marginal change of the scour depth after two and half hours of the experiment and the scour depth reaches the quasiequilibrium state after 3 hours.

\subsection{Pressure gradient of new piggyback pipeline}

To analyze the experimental data, dimensional analysis is carried out. Under the action of steady current, the local scour depth $(h)$ of the piggyback pipeline is mainly related to the following variables: the small pipe diameter $(d)$, the main pipe diameter $(D)$, the specific weight of sediment particles $\left(\gamma_{s}\right)$, the specific weight of water $(\gamma)$, the median diameter of sediment $\left(d_{50}\right)$, the gravity acceleration $(g)$, water viscosity, the current velocity $(v)$ and water depth. In this study, water depth and the sediment median diameter are held as constants. Therefore, the scour depth $(h)$ of new piggyback pipeline can be expressed as:

$$
h=f\left(d, D, \gamma_{s,} \gamma, v, g, v, H, d_{50}\right)
$$


In this study, water depth, the gravity acceleration, the specific weight of sediment particles, the specific weight of water and sediment median diameter are held as constants, their influence on scour depth is therefore not considered. The above equation (4) can be simplified as

$$
h=f(d, D, v, v)
$$

Selecting $v, D, v$ as the basic variables and applying the dimensional analysis yields:

$$
\frac{h}{D}=f\left(\frac{d}{D}, \mathrm{Re}\right)
$$

where $\operatorname{Re}=v D / v$ is the Reynolds number. This shows that under the current tested conditions conducted in this study, the scour depth is mainly related to flow Reynolds number and the ratio between the small and large pipe diameters.

Figure 9 demonstrates the correlation between $R e$ number and pressure gradient ratio at different diameter ratios for $D=10 \mathrm{~cm}$ and $\theta=0.0682(\nu=0.32 \mathrm{~m} / \mathrm{s})$ in which $p_{I}$ is the pressure gradient of the new piggyback pipeline; $p_{0}$ is the pressure gradient of single pipeline. It can be seen from Fig. 9 that the pressure gradient of single main is larger than that of the new piggyback pipe. For the same Reynolds number (e.g. the same incoming flow velocity), the pressure gradient radio decreases with the increase of diameter ratio. This may be ascribed to the fact that the presence of the small pipes upstream and downstream of the large pipe has a shielding effect on the bed surface which reduces the force of the upstream flow on the seabed near the main pipe. Consequently, the pressure gradient between the two sides of the piggyback pipe is reduced. With the increase of the pipe diameter ratio, the shielded area of the bed surface near the main pipe increases, resulting in even small pressure gradient between the two sides of the main pipe.

\subsection{Force coefficients on new piggyback pipeline}


The pressure distribution of different positions of the main pipe is measured using the force transducers installed on the pipeline. The lift force and drag force on the pipeline are calculated by the method of fitting integral as:

$$
\begin{gathered}
F_{d}=-\sum_{n=1}^{12} \frac{\pi D}{12} P_{n} \cos \left(\frac{N \pi}{6}\right) \\
F_{L}=-\sum_{n=1}^{12} \frac{\pi D}{12} P_{n} \sin \left(\frac{N \pi}{6}\right)
\end{gathered}
$$

Where $F_{d}$ is the drag force; $F_{L}$ is the lift force; $N(=12)$ is the number of measurement point; $P_{n}$ is the pressure value measured at the $n$th measurement point. The lift force coefficient and drag force coefficient can then be calculated as:

$$
\begin{gathered}
C_{d}=\frac{2 F_{d}}{\rho D v^{2}} \\
C_{L}=\frac{2 F_{L}}{\rho D v^{2}}
\end{gathered}
$$

Where $v$ is the undisturbed orbital velocity at the horizontal axis of the larger pipeline; $\rho$ is the density of water.

Figure 10 shows the variation of the drag force coefficient (Fig. 10 (a)) and lift force coefficient(Fig. 10 (b)) with time for $D=10 \mathrm{~cm}, R e=32000$ and $\theta=0.068$. The force coefficients for the common piggyback pipeline from Zhao et al. (2018) are also plotted in Fig.10 for comparison. It can be seen from Fig.10 (a), the drag force coefficient on the piggyback pipe decreases gradually with time and is smaller than that on the common piggyback pipeline. When the scour depth reaches the quasi-equilibrium state (e.g. $t>180$ minutes), the variation of the drag force on the piggyback pipe is marginal. This may be explained as following. At the onset of the scour, the scour hole is not yet generated and water flow can only pass over the pipeline. As such, the drag force acting on the bottom of the pipe is small. With the development of scouring, flow through the bottom of main pipeline gradually increases while the wake vortex behind the piggyback pipeline gradually reaches 
steady state. Consequently, the pressure distribution around the pipe has insignificant variation, which results in almost constant drag force coefficient. In addition, as discussed above, the scour depth decreases with the increase of diameter ratio, resulting in a decrease in the drag force. As such, the drag force coefficient decreases with the increase of diameter ratio. When the diameter ratio of the pipe reaches 0.3, the scour depth tends to 0 (as shown in Fig.7 (e)) and the drag force coefficient has marginal change. Fig. 10 (b) demonstrates that the lift force coefficient initially decreases with time and then gradually increases with time. This is because during the initial stage of scouring, the scour hole under pipeline develops with time. As time goes, the scour hole tends to be stable and the force acting on the pipe has insignificant change. It can be seen from Fig.10 (b) that the lift force coefficient around the new pipeline is positive and is higher than that around the common piggyback pipe. This means that the lift force of the pipeline is deviated from the surface of the bed, which prevents the pipeline from sinking. With the increase of the pipe diameter ratio, the lift force coefficient around the new piggyback pipe increases gradually.

\subsection{Equilibrium scour depth}

Figure 11 displays the correlation between Re number and the quasi-equilibrium scour depth for different pipe diameters, in which $h_{1}, h_{2}$ and $h_{3}$ are the equilibrium scour depth for $D=0.08 \mathrm{~m}$, $D=0.1 \mathrm{~m}, D=0.12 \mathrm{~m}$ respectively. The equilibrium scour depth data of single pipe (Zhao (2017); Mao (1986)) and common piggyback pipeline (Zhao et al. (2018); Zhao and Cheng (2008)) are also plotted in Fig.11 for comparison. Fig.11 shows that for the same diameter ratio, the scour depth for typical piggyback pipeline is about $70 \%$ larger than that of the new form piggyback pipeline under the same conditions. Under the same conditions, the scour depth of the new pipe is also smaller than 
that of the single pipe. The scour depth of new piggyback pipe tends to increase with the increase of the flow Re number and decreases with the increase of the diameter ratio. This variation trend is the same as that of the typical piggyback pipeline. This is because a larger Re number is likely to generate greater shear stress at the surface of the bed. But contrary to the typical piggyback pipeline, the scour depth of the new type piggyback pipeline decreases with the increase of the diameter ratio.

\subsection{Equilibrium scour width}

Figure 12 illustrates the effect of Re number on the equilibrium scour width, where $W_{1}, W_{2}$ and $W_{3}$ are the scour width of the quasi-equilibrium sour hole for $D=0.08 \mathrm{~m}, D=0.1 \mathrm{~m}, D=0.12 \mathrm{~m}$, respectively. Similar to the scour depth, the extent of scour hole around single main pipe in greater than that of the new piggyback pipe. Fig. 12 shows that the quasi-equilibrium scour width increases with the increase of $R e$ number and decreases with the increase of the diameter ratio.

\subsection{Numerical results}

Figure 13 shows the comparison of laboratory experimental results and numerical simulation results of velocity distributions in section 2 (Fig. 13(a)); section 4 (Fig. 13(b)) and section 6 (Fig. $13(\mathrm{c}))$ for $d / D=0.25(D=0.1 \mathrm{~m}, d=0.025 \mathrm{~m})$ and $\theta=0.0682$. It is seen from Fig. 13 that the simulated velocity field in general agrees well with the measurements though some discrepancy between simulation and experiment exists at section 4 where the vortex is generated. This indicates that the numerical model has capacity to accurately reproduce the complex flow field around the piggyback pipeline.

Figure 14 shows the comparison of simulated vertical velocity profiles at different sections for 
single pipe, typical piggyback pipeline and new type piggyback pipeline for $D=0.1 \mathrm{~m}, d=0.025 \mathrm{~m}$, $R e=32000$ and $\theta=0.0682$. Fig. 14 shows that in general, the vertical velocity profiles for new piggyback pipes are similar to those for single pipe. Slight difference of the velocity profile near the bed in the wake region is noticed, indicating that the small pipe in the piggyback pipelines has certain effect on the flow field near bed. Fig.14 (a), (b) and (e) shows that the vertical velocity profiles of the three different pipeline arrangements are similar at the far upstream section 1 and section 2 and far downstream section 6 and follow the traditional exponential distribution. This indicates that the presence of pipelines has insignificant effect on the flow velocity far upstream of the pipeline. It is seen from Fig 14 (c) that the velocity profile at the directly above pipeline section 3 for typical piggyback pipeline is larger than those for the single and new piggyback pipelines. This is because the obstruction effect from the typical piggyback pipeline is larger than that for single and new piggyback pipelines, leading to the increase of velocity for the typical piggyback pipelines. The velocities of section 4 and 5 are shown in Fig 14(d) and (e). The two sections are located in the wake region and the reverse velocities at these two sections indicate that vortices are generated in both sections for all pipeline arrangements. Comparing Fig 14 (d) and (e), it can be seen that the intensity of vortex in section 5 is less than that in section 4. Fig.14 (d) and (e) also show that the vortex strength of the typical piggyback pipeline is larger than that for single and new piggyback pipeline due to the greater blockage effect from the former piggyback pipeline.

\section{Conclusions}

In this study, the flow field and pressure distribution, the force coefficients, the quasiequilibrium scour extent (e.g. depth and width) of the new piggyback pipeline have been 
investigated for a range of parameters, including pipe diameter ratio and velocity using numerical simulation and physical model experiments. From the analysis of the results, the main conclusions of this study are:

1) The new piggyback pipeline configuration can effectively reduce the pressure gradient between the upstream and downstream side of the pipeline, which can in turn reduce the local scour around the pipeline. The pressure gradient of the new piggyback pipeline decreases with the increase of the pipe diameter ratio. The strength of wake vortex is smaller than that of the typical piggyback pipeline under the same conditions.

2) For the parameters tested in this study, the quasi-equilibrium scour extent (e.g. depth and width) around the new piggyback pipeline is smaller than that of typical piggyback pipeline for the same pipe diameter ratio under the same conditions. Both the quasi-equilibrium scour depth and width increase with the increase of $R e$ number and decrease with the increase of the pipe diameter ratio.

\section{Acknowledgments}

This work is supported by the National Natural Science Foundation of China (No. 51279189).The writers would like to thank Mr. Baocheng Kan for his help in the experiment. Comments made by reviewers have greatly improved the quality of the paper.

\section{References}

Brockbank, J.,1990. Bundled pipe speed offshore laying, Oil and Gas J. 88(19), 78-84. 
Cevik, E., Yüksel, Y., 1999. Scour under submarine pipelines in waves in shoaling conditions. Journal of Waterway, Port, Coastal, and Ocean Engineering, ASCE, 125 (1), 9-19.

Cheng, X.F., Wang, Y.X., Wang, G.Y., 2013. Influence of proximity of the seabed on hydrodynamic forces on a submarine piggyback pipeline under wave action. J. Offshore Mechanics and Arctic Eng. 135(2), 021701.

Cheng, X.F., Wang, Y.X., Wang, G.Y., 2012. Physical model experimental study of hydrodynamic forces on submarine piggyback pipeline under wave-current coexisting action. Acta Oceanological Sinica.34(4), 172-180.

Chien, N., Wan, Z.H., 1999. Mechanics of sediment transport. ASCE Press, New York.

Einstein, H.A., Chien, N., 1955. Effects of heavy sediment concentration near the bed on the velocity and sediment distribution. University of California, USA.

Fredsøe, J. 2016. Pipeline-seabed interaction, Journal of Waterway, Port, Coastal and Ocean Engineering, ASCE, 142(6), 03116002.

Guo, Y.K., Zhang, L.X., Shen, Y.M. and Zhang, J.S., 2008. Modelling study of free overfall in a rectangle channel with strip roughness, Journal of Hydraulic Engineering (ASCE), 134(5): 664-668.

Guo, Y.K., Wu, X.G., Pan, C.H. and Zhang, J.S. 2012. Numerical simulation of the tidal flow and suspended sediment transport in the Qiantang Estuary. ASCE J. Waterway, Port, Coastal and Ocean Eng., 138(3), 192-203.

Guo, Y.K., Zhang, Z.Y. and Shi, B. 2014. Numerical simulation of gravity current descending a slope into a linearly stratified environment. Journal of Hydraulic Engineering (ASCE), 140(12) DOI: 10.1061/(ASCE)HY.1943-7900.0000936. 
Jakobsen, M.L., Sayer, P., 1995. Hydrodynamic forces on piggyback pipelines. The 5th International Offshore and Polar Engineering Conference, Hague, Netherlands, 1, 139-147.

Jester, W., Kallinderis, Y.,2003. Numerical study of incompressible flow about fixed cylinder pair. J. Fluids and Structures. 17(4), 561-577.

Jing, H., Guo, Y.K., Li, C. and Zhang, J. 2009. Three dimensional numerical simulation of compound meandering open channel flow by Reynolds stress equation model. International Journal for Numerical Methods in Fluids, 59:927-943.

Jing, H., Li, C., Guo, Y.K. and Xu, W.L. 2011. Numerical simulation of turbulent flows in trapezoidal meandering compound open channels. International Journal for Numerical Methods in Fluids, 65:1071-1083.

Kalghatgi, S. G., Sayer, P. G.,1997. Hydrodynamic forces on piggyback pipelines configurations. J. Waterway, Port, Coastal and Ocean Eng.123, 16-22.

Kamarudin,M.H., Thiagarajan,K.P., Czajko, A., 2006. Analysis of current-induced force on offshore pipeline bundles. Proceedings of the $5^{\text {th }}$ International Conferenceon CFD in the Process Industries, Melbourne, Australia,Vol.1, pp. 1-6.

Launder, B.E., and Spalding, D.B. 1974. The numerical computation of turbulent flows. Computer Methods in Applied Mechanics and Engineering, 3,269-289.

Lee, K., Yang, K.S., 2009. Flow patterns past two circular cylinders in proximity. Computers \&Fluids. 38(4), 778-788.

Lin, Z.B., Guo, Y.K., Jeng, D.S., Liao, C.C. and Rey, N. 2016. An integrated numerical model for wave-soil-pipeline interactions. Coastal Engineering, 108: 25-35. 
Lin, Z.B., Pokrajac, D., Guo, Y.K., Jeng, D.S., Tang, T., Rey, N., Zheng, J.H., Zhang, J.S. 2017. Investigation of nonlinear wave-induced seabed response around mono-pile foundation. Coastal Engineering, 121: 197-211

Liu, K., Ma, D.J., Sun, D.J., Yin, X.Y.,2007. Wake patterns of flow past a pair of circular cylinders in side-by-side arrangements at low Reynolds numbers. J. Hydrodynamics. 19(6), 690-697.

Ma, L., Wang, J.Y., Sun, S.S., Xing, Z.Z., 1993. Experimental study of hydrodynamic force loading under the action of wave current on submarine composite pipelines. J. Dalian University. 3(4), $55-63$.

MacPherson, H. 1978. Wave forces on pipeline buried in permeable seabed, Journal of Waterway, Port, Coastal Ocean Division, ASCE, 104, 407-419.

Mao, Y.,1986. The interaction between a pipeline and an erodible bed ( $\mathrm{PhD}$ thesis). Technical University of Denmark, Denmark.

Najafzadeh M., Barani, G-A, Azamathulla, H.M., 2014a. Prediction of pipeline scour depth in clearwater and live-bed conditions using group method of data handling, Neural Computing and Applications. 24: 629-635.

Najafzadeh M., Barani, G-A, Hessami-Kermani, M. 2014b. Estimation of Pipeline Scour due to Waves by GMDH. Journal of Pipeline Systems Engineering and Practice. ASCE 5(3) 06014002. Najafzadeh, M., Sarkamaryan, S., 2018. Extraction of optimal equations for evaluation of pipeline scour depth due to currents. ICE Maritime Engineering, 171(1): 1-10.

Najafzadeh, M. and Saberi-Movahed, F. 2018. GMDH-GEP to predict free span expansion rates below pipelines under waves. Marine Georesource and Geotechnology, published online 12 March 2018. 
Pan, P., Cai, B.Y., Tan, A.L., Guo, C.Y., Xie, S.H., 2015. Research on vortex induced vibration of cable in piggyback configuration. Ocean Eng. 33(6), 45-51.

Sudhan, C.M., Sundar, V., Rao, S. N. 2002. Wave induced forces around buried pipelines, Ocean Engineering, 29(5), 533-544.

Sui, T.T., Zhang, C., Guo, Y.K., Zheng, J.H., Jeng, D.S., Zhang, J.S., and Zhang, W. 2016. Threedimensional numerical model for wave-induced seabed response around mono-pile. Ships and Offshore Structures, 11(6), 667-678

Sui, T., Zheng, J.H., Zhang, C., Jeng, D.S., Zhang, J.S., Guo, Y.K., He, R. 2017. Consolidation of unsaturated seabed around an inserted pile foundation and its effects on the wave-induced momentary liquefaction. Ocean Engineering, 131: 308-321

Sumer, B.M., Truelsen, C., Sichmann, T. and Fredsøe, J., 2001. Onset of scour below pipelines and self-burial. Coast Engineering 42, 313-335.

Sumer, B. M. 2014. Flow-structure-seabed interactions in coastal and marine environments, Journal of Hydraulic Research, 52(1), 1-13.

Sun, K., Zhang, J.S., Gao, Y., Jeng, D.S., Guo, Y.K. and Liang, Z.D. 2019. Laboratory experimental study of ocean waves propagating over a partially buried pipeline in a trench layer. Ocean Engineering, 173: 617-627.

Wilcox, D.C. 2008. Formulation of the k-omega Turbulence Model Revisited. AIAA Journal, 46(11), 2823-2838.

Yang, H., Ni, H., Zhu, X.H.,2007. An applicable replacement bundled pipeline structure for offshore marginal oilfield development. Ship Building of China.48, 563-570. 
Yang, L.P., Guo, Y.K., Shi, B., Kuang, C.P., Xu, W.L., Cao, S.Y.,2012. Study of scour around submarine pipeline with a rubber plate or rigid spoiler in wave conditions. J. Waterway, Port, Ocean and Coast Eng, 138 (6):484-490.

Yang, L.P., Shi, B., Guo, Y.K., Wen, X.Y.,2012. Calculation and experiment on scour depth for submarine pipeline with a spoiler, Ocean Eng, 55:191-198.

Yang, L.P., Shi, B., Guo, Y.K., Zhang, L.X., Zhang, J.S., Han Y.,2014. Scour protection of submarine pipelines using rubber plates underneath the pipes. Ocean Eng, 84, 176-182.

Zang, Z.P., Gao, F.P., Cui, J.S.,2013.Physical modeling and swirling strength analysis of vortex shedding from near-bed piggyback pipelines. Appl. Ocean Res. 40(1), 50-59.

Zang, Z.P., Gao, F.P.,2014. Steady current induced vibration of near-bed piggyback pipelines: configuration effects on VIV suppression. Appl. Ocean Res. 46(2), 62-69.

Zhang, Z.Y., Shi, B., Guo, Y.K., Yang, L.P.,2013. Numerical investigation on critical length of impermeable plate below underwater pipeline under steady current. Science China (Tech Sciences). 56, 1232-1240.

Zhao, M., Cheng, L., Teng, B., 2007. Numerical modelling of flow and hydrodynamic forces around a piggyback pipeline near the seabed. J. Waterway, Port, Coastal, and Ocean Eng. 133(4),286295.

Zhao, M., Cheng, L., 2008. Numerical modelling of local scour below a piggyback pipeline in currents. J. Hydraul. Eng. 134 (10), 1452-1463.

Zhao, M.,2012. Influence of the position angel of the small pipeline on vortex shedding flow around a sub-sea piggyback pipeline. Coast. Eng. 54(3),117-125. 
Zhao, E.J., Shi, B., Qu, K., Dong, W.B., Zhang, J.,2018. Experimental and numerical investigation of local scour around submarine piggyback pipeline under steady currents. J. Ocean University of China. 17(2), 244-256.

Zhao. E.J.,2017. Study on the dynamic process of scour and seabed evolution around submarine piggyback pipeline (PhD thesis). Ocean University of China, China.

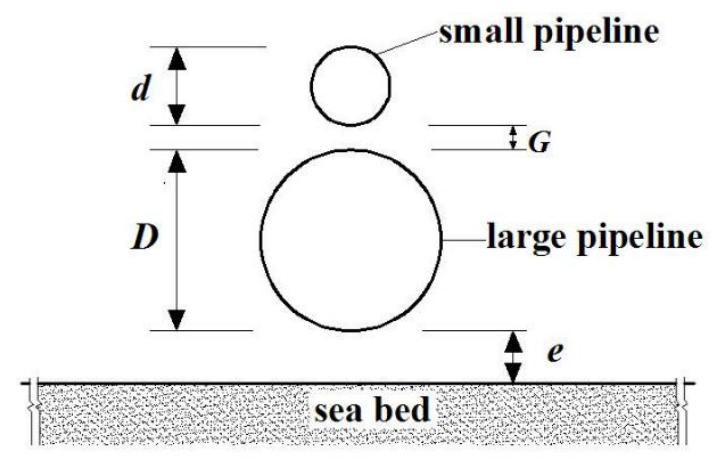

(a)

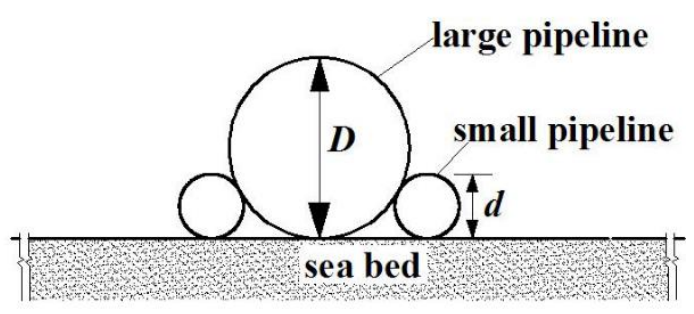

(b)

Fig 1

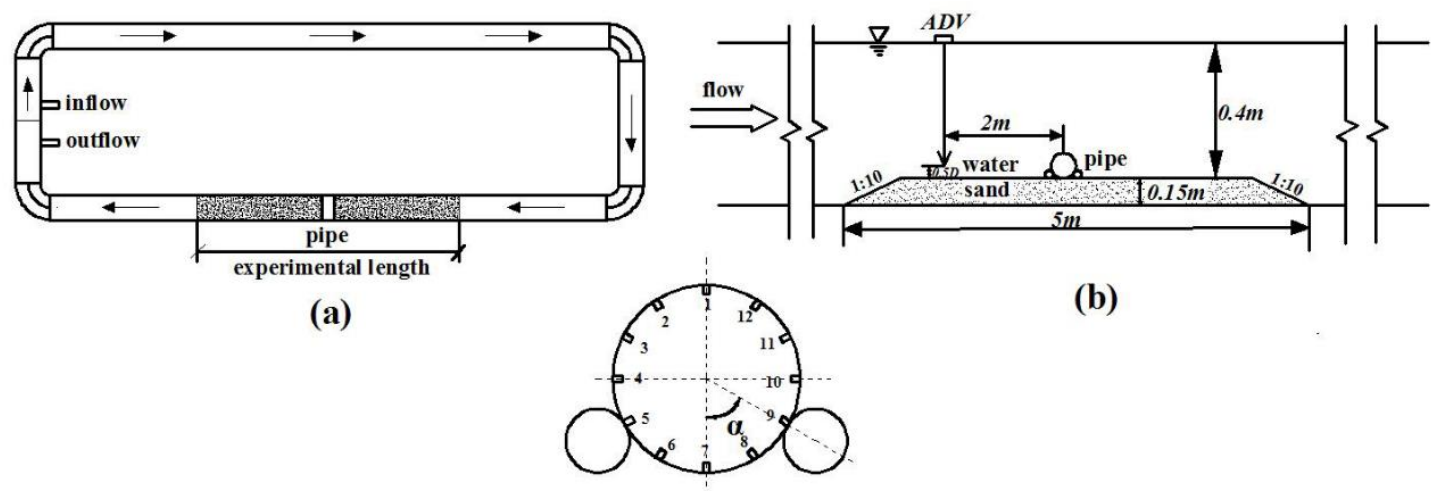

(c)

Fig 2 


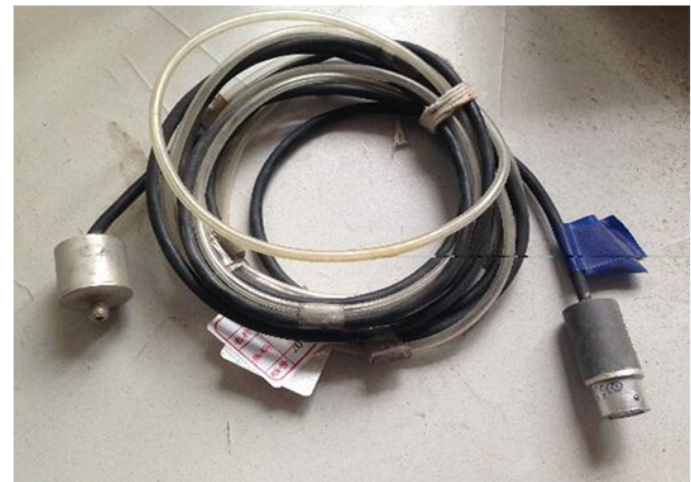

(a) force transducer

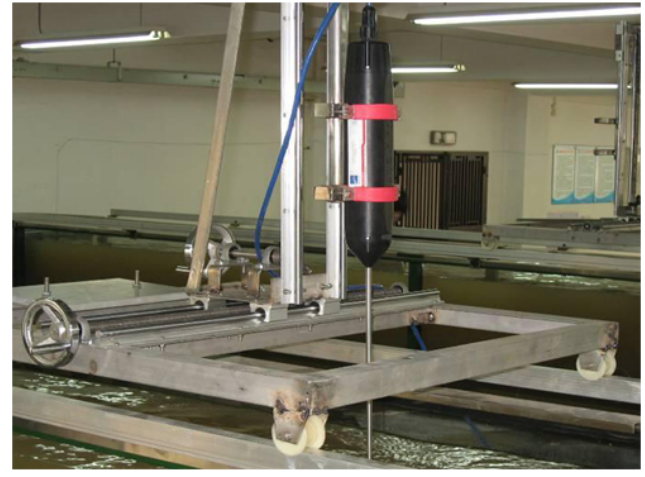

(b) ADV

Fig 3

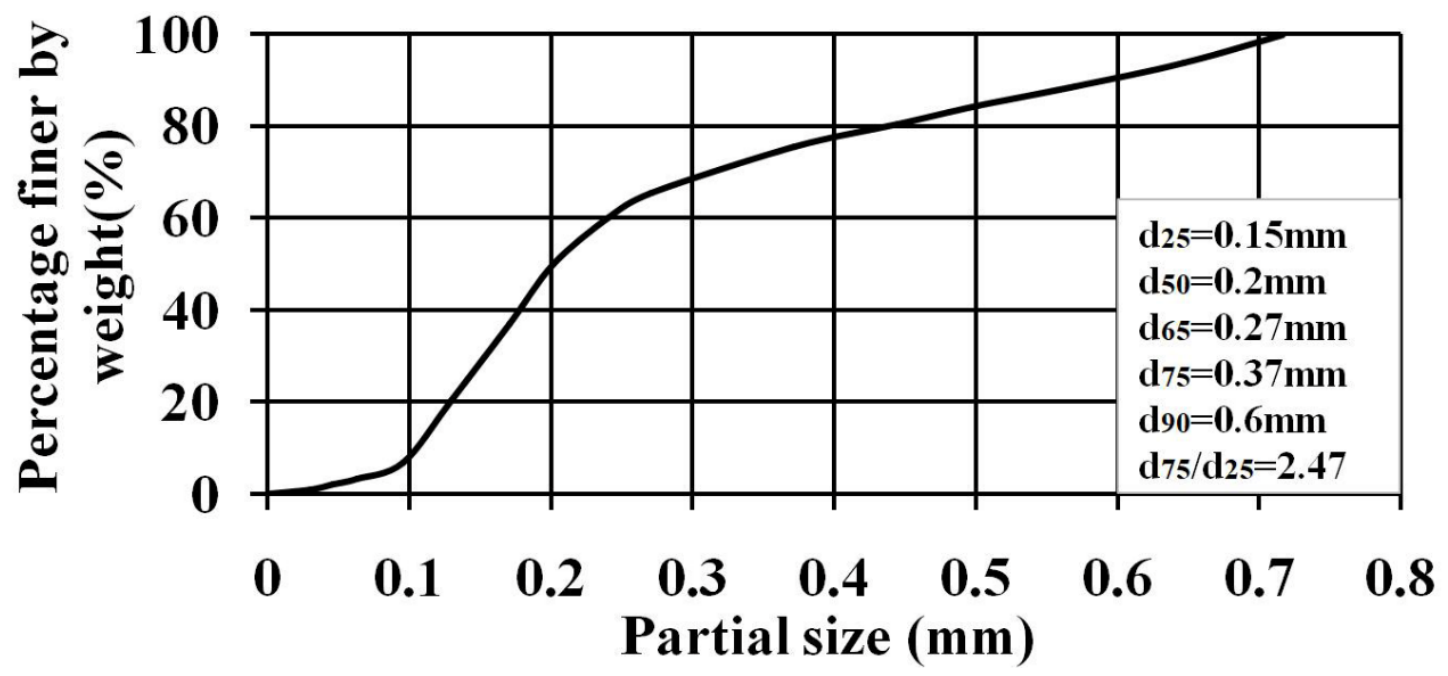

Fig. 4 


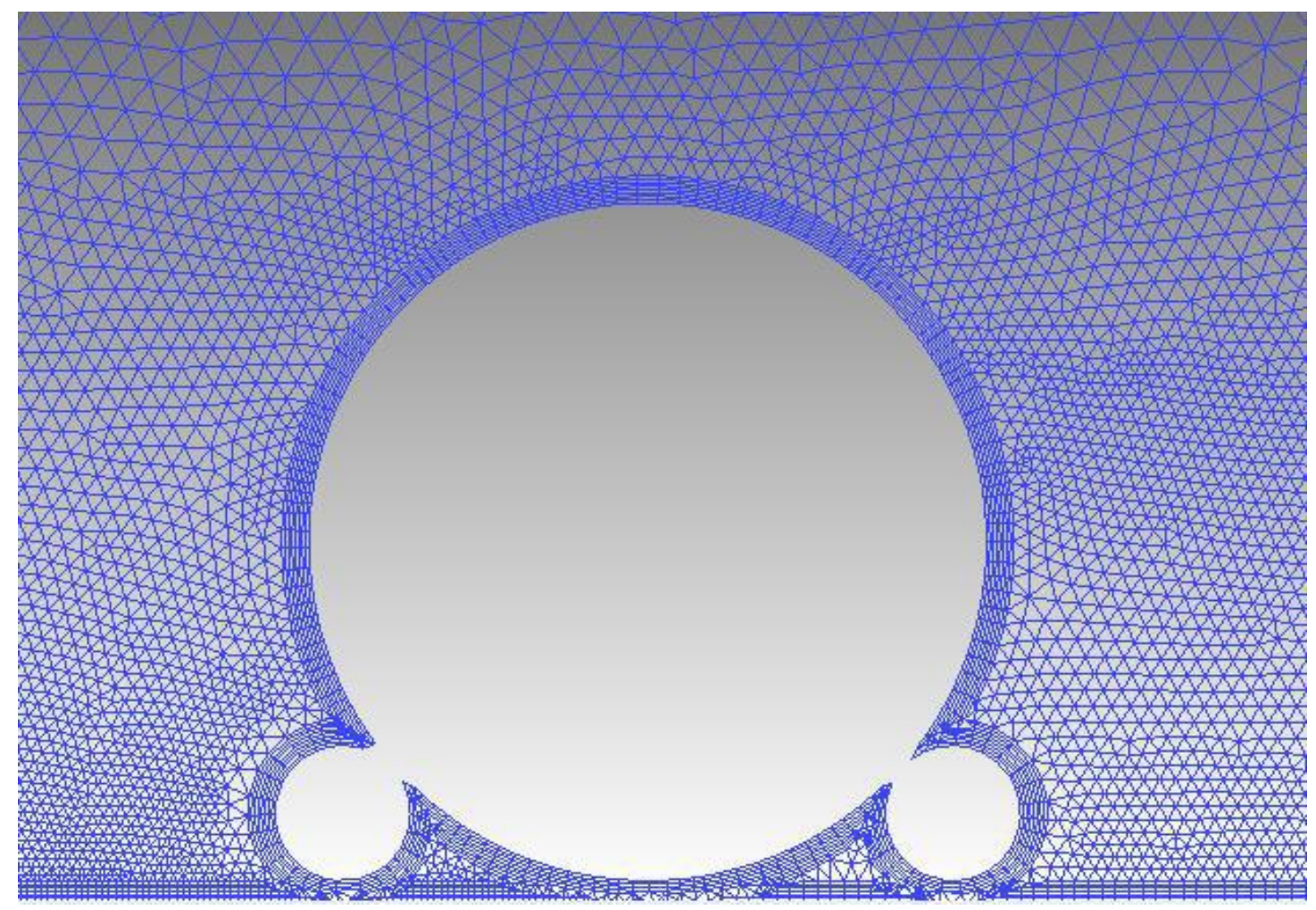

Fig. 5

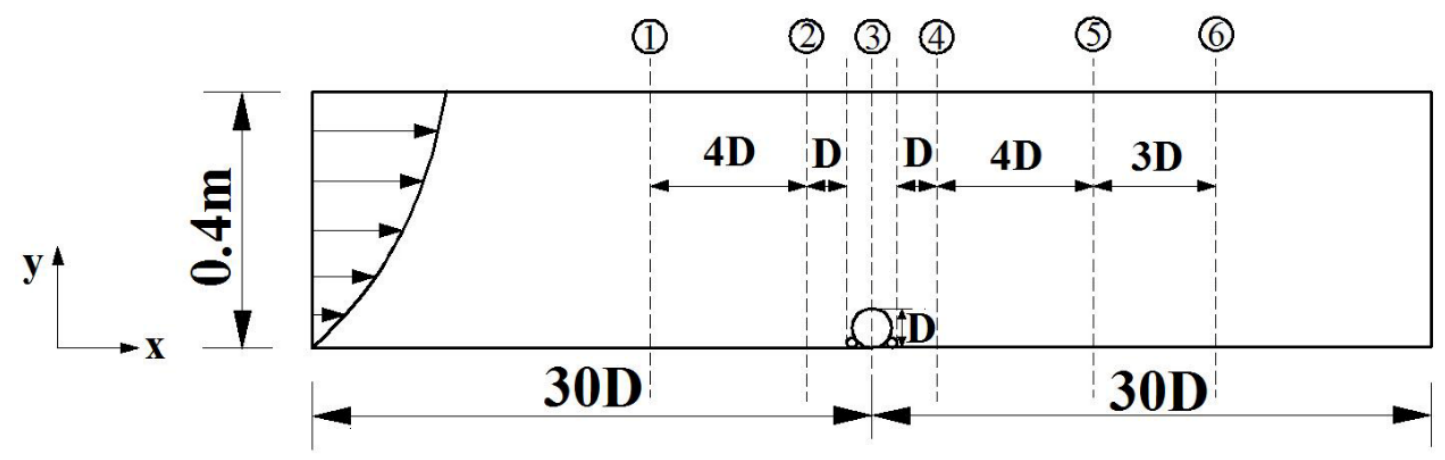

Fig6 


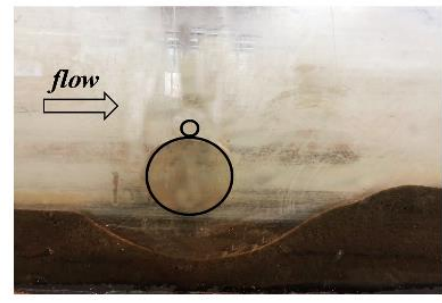

(a) $d=0.02 \mathrm{~m} \mathrm{D}=0.1 \mathrm{~m}$

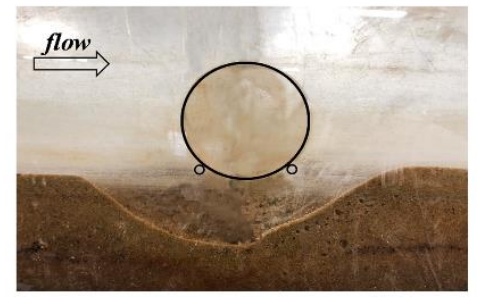

(b) $\mathrm{d}=0.01 \mathrm{~m} \mathrm{D}=0.1 \mathrm{~m}$

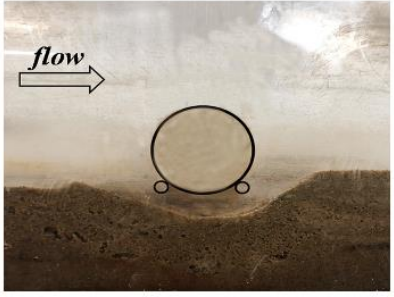

(c) $\mathrm{d}=0.02 \mathrm{~m} \mathrm{D}=0.1 \mathrm{~m}$

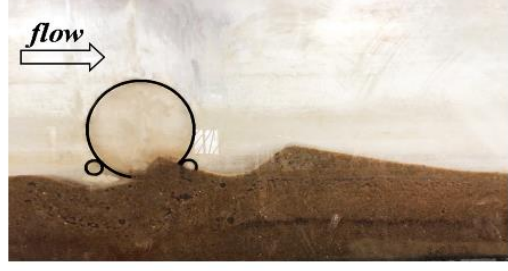

(d) $d=0.025 \mathrm{~m} \mathrm{D}=0.1 \mathrm{~m}$

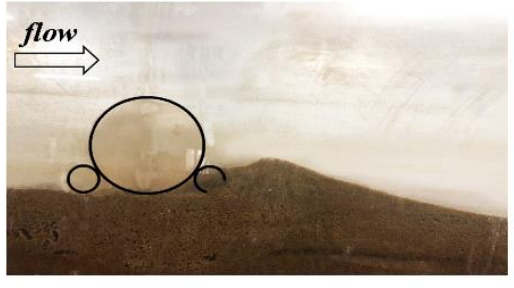

(e) $d=0.03 \mathrm{~m} \mathrm{D}=0.1 \mathrm{~m}$

Fig7

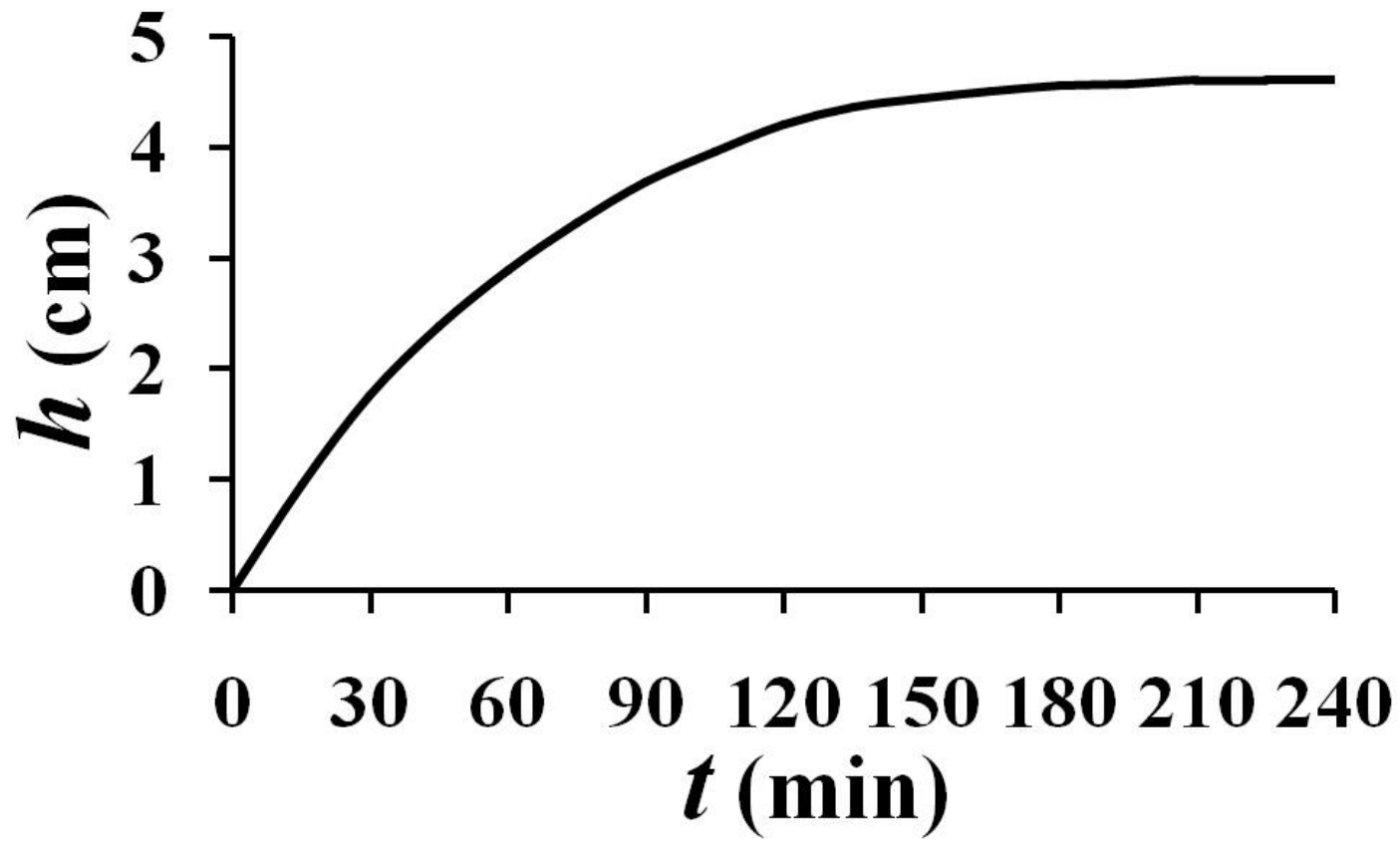

Fig. 8 


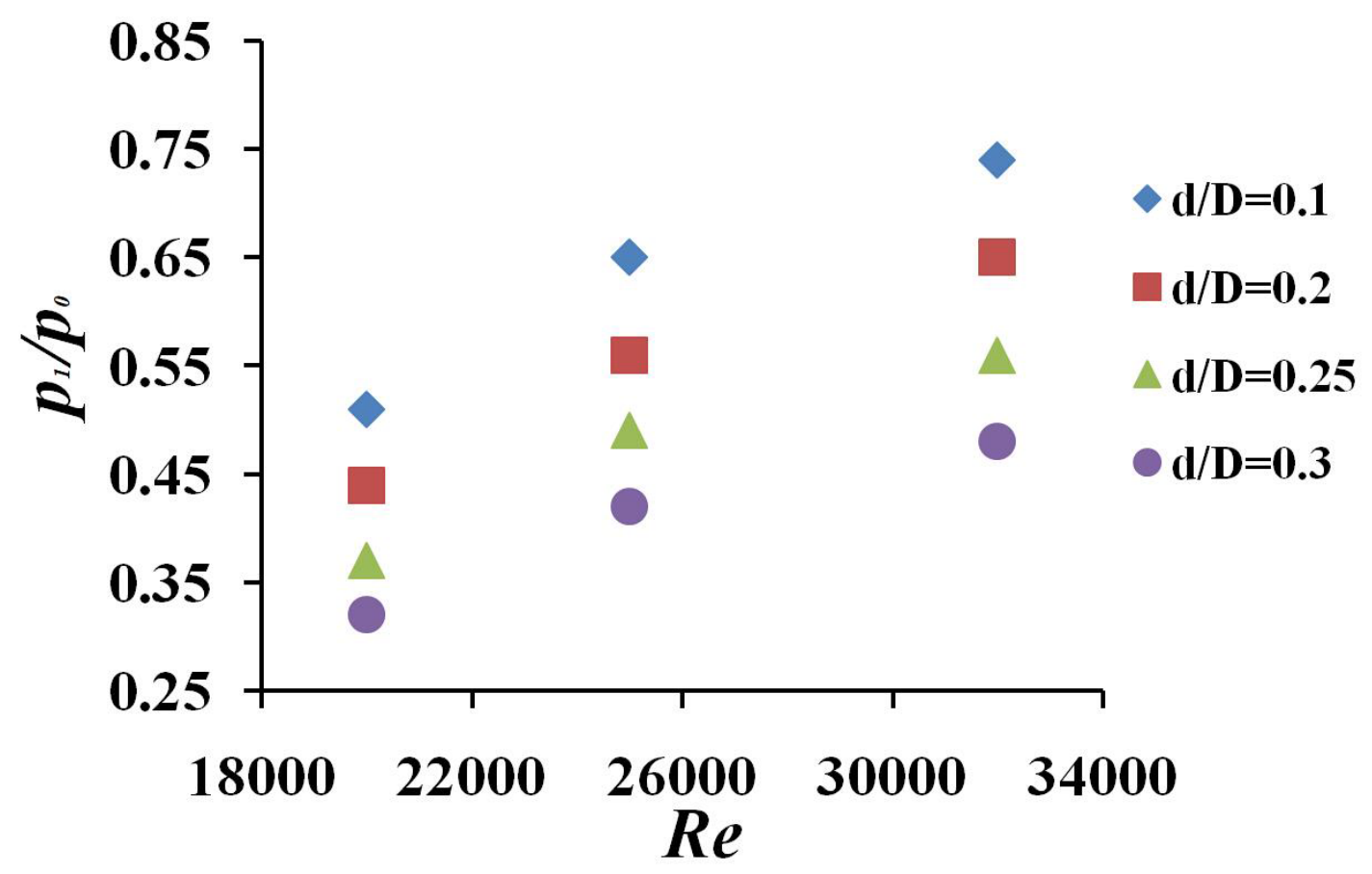

Fig.9

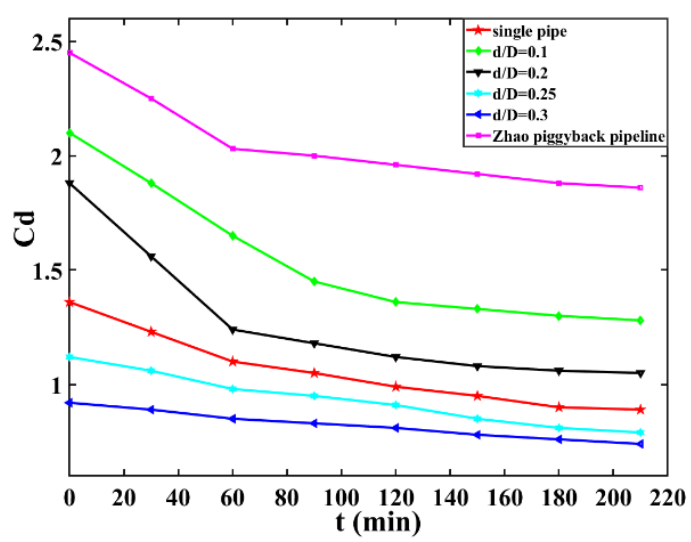

(a) Drag force coefficient

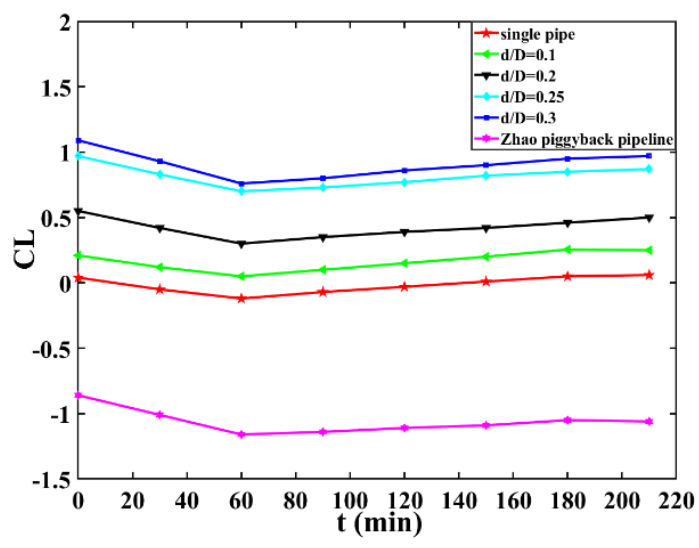

(b) Lift force coefficient

Fig. 10 


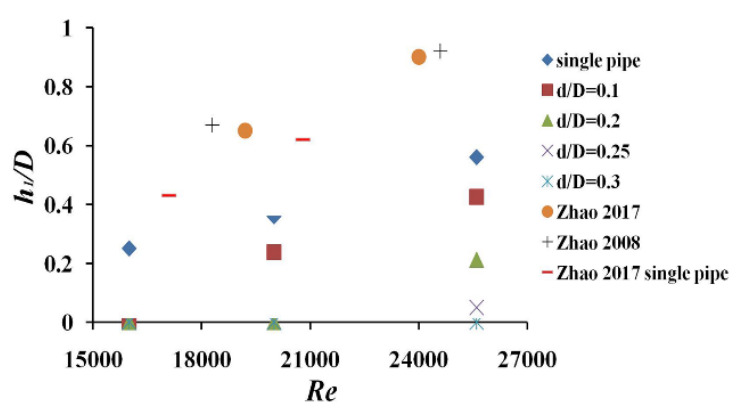

(a) $\mathrm{D}=0.08 \mathrm{~m}$

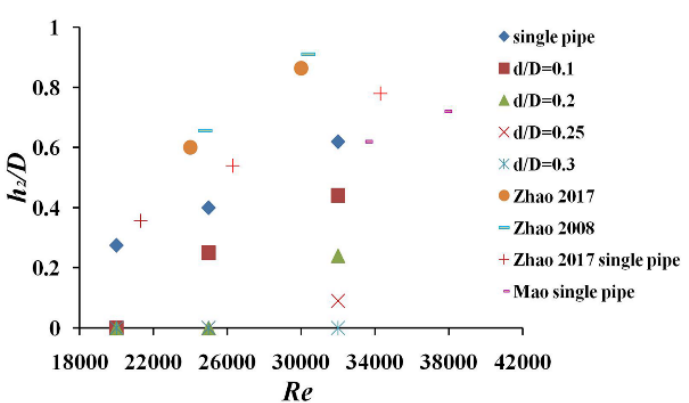

(b) $\mathrm{D}=0.1 \mathrm{~m}$

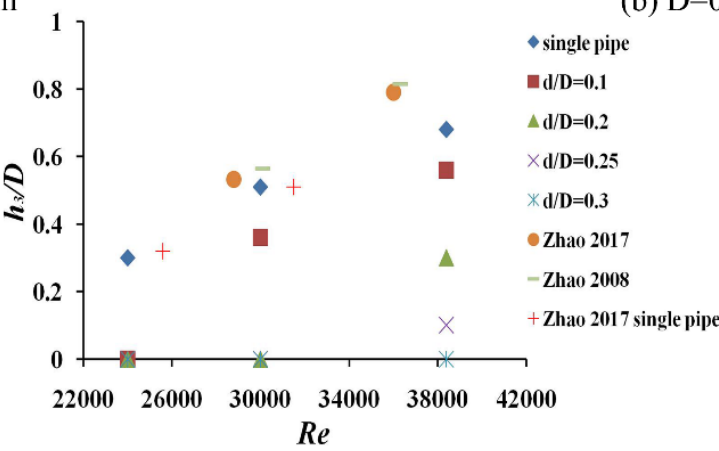

(c) $\mathrm{D}=0.12 \mathrm{~m}$

Fig. 11

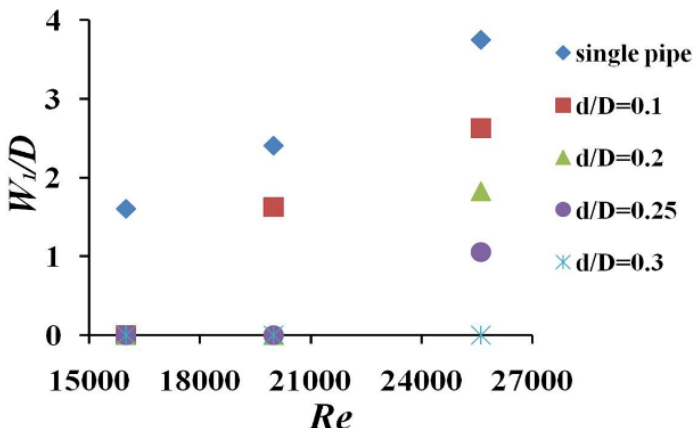

(a) $\mathrm{D}=0.08 \mathrm{~m}$

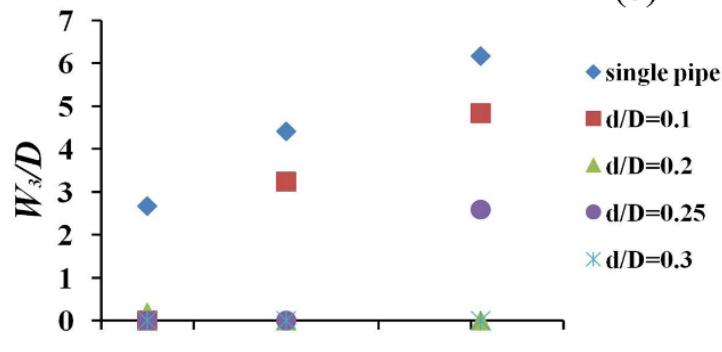

220002600030000340003800042000

Re

(c) $\mathrm{D}=0.12 \mathrm{~m}$

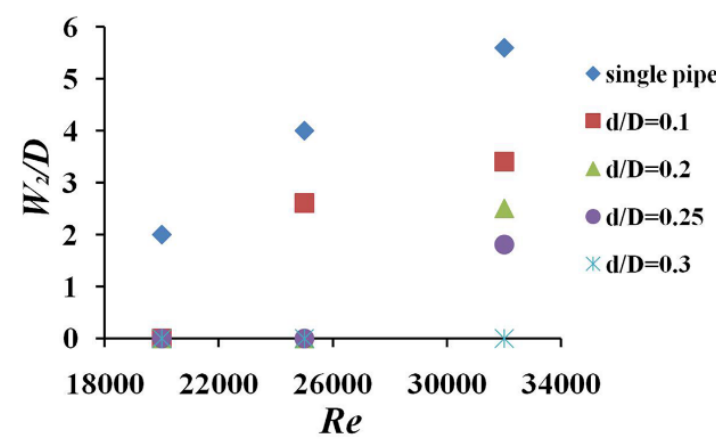

(b) $\mathrm{D}=0.1 \mathrm{~m}$

Fig. 12 


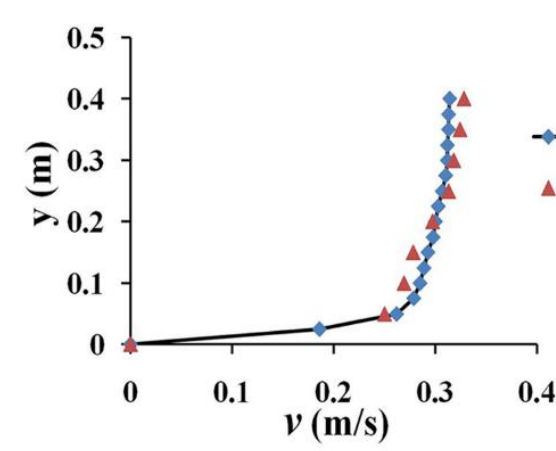

(a) Section 2

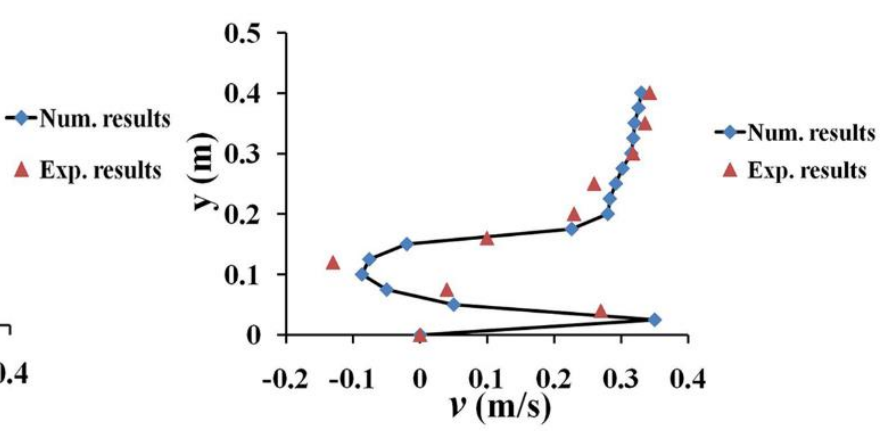

(b) Section 4

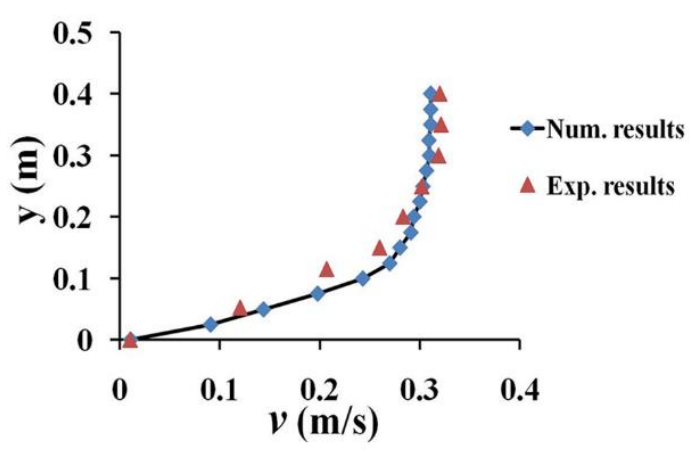

(c) Section 6

Fig13

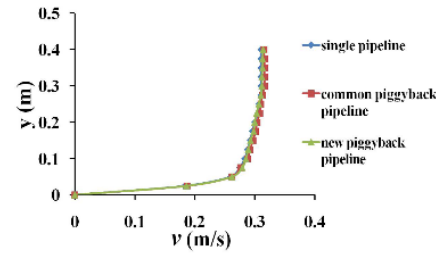

(a)

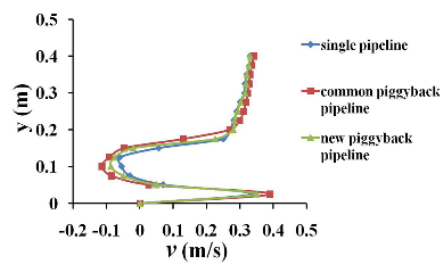

(d)

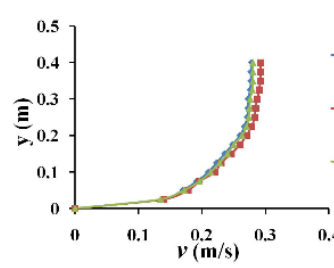

(b)

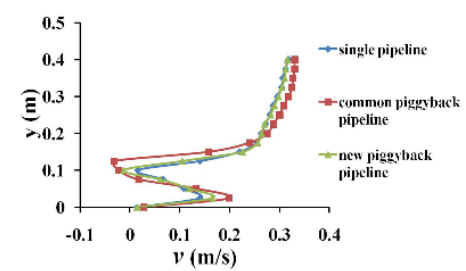

(e)

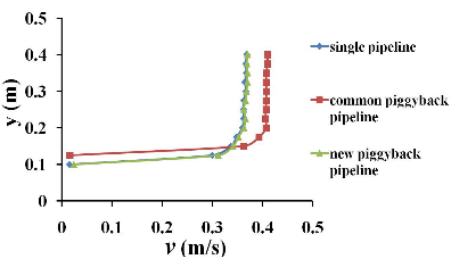

(c)

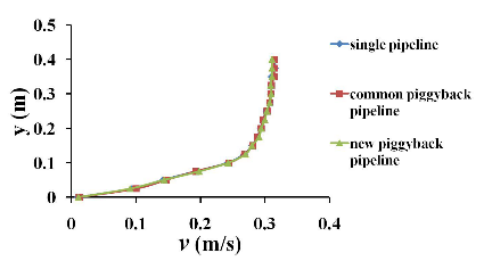

(f)

Fig14

\section{Figure captions}

Fig.1.Schematic diagram submarine piggyback pipeline configuration

Fig.2. Sketch of the experimental layout

Fig.3. Photo showing the experimental measurement equipments

Fig.4. Particle size distribution curve of the sand tested 
Fig.5. Computational mesh near the piggyback pipeline

Fig.6. Locations showing the vertical velocity profiles taken from the numerical simulation

Fig.7 Experimental photos showing the sediment transport and scour around various piggyback pipeline arrangements

Fig. 8 . Variation of the scour depth (h) with time ( $\mathrm{t}$ ) for $\mathrm{d}=0.01 \mathrm{~m} \mathrm{D}=0.1 \mathrm{~m}, R e=32000$ and $\theta=0.0682$

Fig.9.Variation of pressure gradient radio with Re number for $\mathrm{D}=0.1 \mathrm{~m}$

Fig. 10. Force coefficients on the new piggyback pipeline for $\mathrm{D}=0.1 \mathrm{~m}, R e=32000$ and $\theta=0.0682$

Fig.11. Variation of equilibrium scour depth with Re number for different diameter ratios

Fig.12. Variation of equilibrium scour width with Re number for different diameter ratios

Fig.13. Comparison of experimental results and numerical simulation results for velocity distributions in different sections

Fig.14. Comparison of simulated vertical velocity profiles for single pipe, typical piggyback pipeline (see Fig.1 (a)) and new type piggyback pipeline (see Fig. 1 (b)) for $\mathrm{D}=0.1 \mathrm{~m}, \mathrm{~d}=0.025 \mathrm{~m}$, $R e=32000$ and $\theta=0.0682$.

Table1. Experimental parameters*

\begin{tabular}{lcccr}
\hline Run & \multicolumn{1}{c}{$D(\mathrm{~m})$} & $d(\mathrm{~m})$ & diameter ratio $(d / D)$ & $v(\mathrm{~m} / \mathrm{s})$ \\
\hline 1 & $0.08 / 0.1 / 0.12$ & 0 & 0 & $0.2 / 0.25 / 0.32$ \\
2 & 0.08 & $0.008 / 0.016 / 0.02 / 0.024$ & $0.1,0.2,0.25,0.3$ & $0.2 / 0.25 / 0.32$ \\
3 & 0.1 & $0.01 / 0.02 / 0.025 / 0.03$ & $0.1,0.2,0.25,0.3$ & $0.2 / 0.25 / 0.32$ \\
4 & 0.12 & $0.012 / 0.024 / 0.03 / 0.036$ & $0.1,0.2,0.25,0.3$ & $0.2 / 0.25 / 0.32$ \\
\hline
\end{tabular}

*: $D=$ main pipe diameter; $d=$ small pipe diameter; $v=$ incoming flow velocity 


\section{List of symbols}

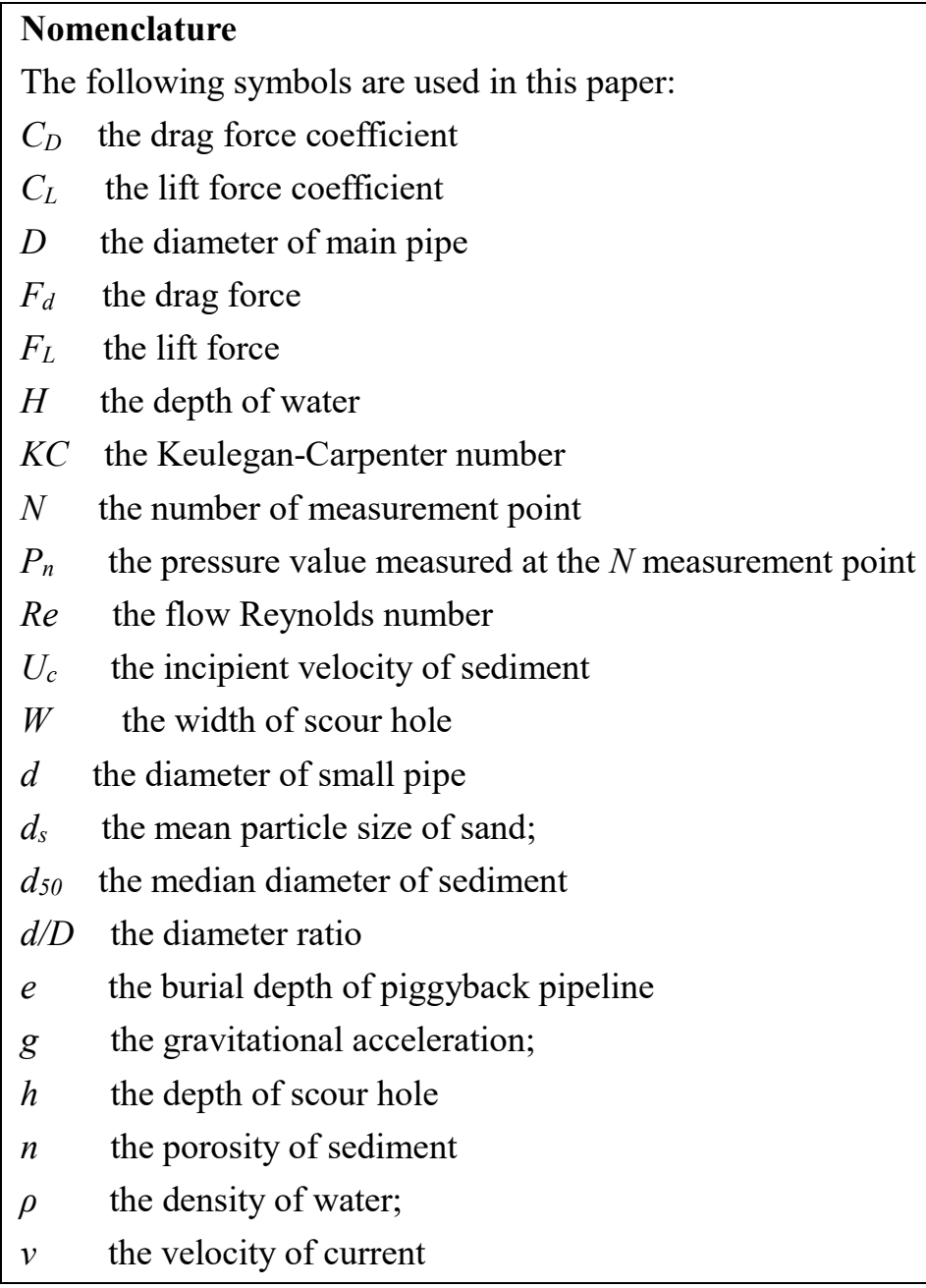

The following symbols are used in this paper:

$C_{D}$ the drag force coefficient

$C_{L}$ the lift force coefficient

$D \quad$ the diameter of main pipe

$F_{d}$ the drag force

$F_{L} \quad$ the lift force

$H$ the depth of water

$K C$ the Keulegan-Carpenter number

$N \quad$ the number of measurement point

$P_{n} \quad$ the pressure value measured at the $N$ measurement point

$R e$ the flow Reynolds number

$U_{c} \quad$ the incipient velocity of sediment

$W \quad$ the width of scour hole

$d \quad$ the diameter of small pipe

$d_{s}$ the mean particle size of sand;

$d_{50}$ the median diameter of sediment

$d / D$ the diameter ratio

$e \quad$ the burial depth of piggyback pipeline

$g$ the gravitational acceleration;

$h \quad$ the depth of scour hole

$n \quad$ the porosity of sediment

$\rho \quad$ the density of water;

$v \quad$ the velocity of current 